\section{mBtd is required to maintain signaling during murine limb development}

\author{
Dieter Treichel, ${ }^{1,3}$ Frieder Schöck, ${ }^{2}$ \\ Herbert Jäckle, ${ }^{2}$ Peter Gruss, ${ }^{1}$ and \\ Ahmed Mansouri,4 \\ ${ }^{1}$ MPI für biophysikalische Chemie, Abt. Molekulare \\ Zellbiologie, Am Fassberg, 37077 Göttingen, Germany; \\ ${ }^{2}$ MPI für biophysikalische Chemie, Abt. Molekulare \\ Entwicklungsbiologie, Am Fassberg, 37077 Göttingen, \\ Germany
}

buttonhead (btd) encodes an SP1-like transcription factor required for the generation and specification of Drosophila head segments. We identified a murine btd homolog, termed mouse Btd (mBtd), which can support btd-dependent head development in transgenic fly embryos. Functional studies show that $\mathrm{mBtd}$-deficient mice develop to term and die at birth. They exhibit brain malformations, posterior axial skeleton truncations, and shortened limbs. We present evidence that $\mathrm{mBtd}$ is required during early limb development to maintain, but not to initiate Wnt/ $\beta$-catenin-dependent FGF, Shh, and BMP-mediated signaling. The data indicate that $\mathbf{m B t d}$ represents a novel key player mediating proximodistal outgrowth of the limb.

Supplemental material is available at http://www.genesdev.org.

Received May 30, 2003; revised version accepted September 5, 2003.

buttonhead (btd), empty spiracles (ems), and orthodenticles (otd) are required for anterior head development during Drosophila embryogenesis (Cohen and Jürgens 1991; Finkelstein and Boncinelli 1994). The three genes are expressed in overlapping domains at blastoderm stage. $b t d$ is required for development of the mandibular, btd and ems for the intercalary, and the combination of $b t d, e m s$, and otd for the antennal segment. ems and otx encode homeodomain transcription factors, and btd encodes an Egr-like zinc finger transcription factor (Wimmer et al. 1993).

Homologs of ems and otd have been identified in mouse. Functional analysis revealed that they are required for patterning processes during early embryonic brain development (Simeone 2002). A vertebrate homo$\log$ of $b t d$ has not yet been identified. However, sequence-related genes with a high degree of similarity were isolated. This includes human and mouse Sp1

[Keywords: FGF signaling; buttonhead; mBtd; limb development; mouse mutant]

${ }^{3}$ Present address: Max-Planck-Gesellschaft, Hofgartenstr. 8, 80539 München, Germany.

${ }^{4}$ Corresponding author.

E-MAIL amansou@gwdg.de; FAX (551) 201-1504.

Article and publication are at http://www.genesdev.org/cgi/doi/10.1101/ gad.274103.
(Wimmer et al. 1993; Schöck et al. 1999a) and the btdlike gene bts1 of zebrafish (Tallafuss et al. 2001). Furthermore, BTD and SP1 proteins are capable of binding to the same DNA sites in vitro, share most biochemical properties, and regulate transcription via the same interacting factors (Wimmer et al. 1993; Schöck et al. 1999a). However, transgenes expressing $S p 1$ or bts 1 in place of $b t d$ failed to rescue $b t d$ mutant embryos. This finding implies that despite the molecular similarities, none of the known vertebrate $b t d$-like genes represents a $b t d$ ortholog (Schöck et al. 1999b; Tallafuss et al. 2001).

Here we report the identification and the functional analysis of a murine btd homolog, termed mouse Btd $(m B t d)$, showing that its activity rescues head segment formation in btd mutant fly embryos. Loss of $m B t d$ in the mouse embryo causes severe brain defects and truncations of body structures, including the posterior axial skeleton and limbs.

We focused our attention on the requirement of $m B t d$ activity on limb development, a process that is initiated through interactions between ectoderm and mesenchyme. The result of these interactions is the formation of the so-called apical ectodermal ridge (AER), a signaling center which is generated through the concerted signaling activities of fibroblast growth factors (FGFs) and Wnt/ $\beta$-catenin (Tickle 2003). We show that the initial generation of the AER by Wnt3 is not affected in mBtd mutants, whereas the expression of Fgfs and limb patterning genes is altered. Lack of $m B t d$ results in apoptotic cell death in both mesenchymal and ectodermal tissue of the limb bud. The data provide evidence that the transcription factor $m B t d$ is required to maintain gene expression initiated by FGF and Wnt signaling necessary for proximodistal limb outgrowth.

\section{Results and Discussion}

The btd homolog was isolated by a PCR-based approach, using degenerated primer pairs within the $b t d$ zinc finger box (Treichel et al. 2001). A resulting 230-bp DNA fragment was subsequently used to screen an embryonic day (E)8.5 mouse cDNA library. Several overlapping cDNA clones were isolated which represent the entire coding sequence of the btd-like gene.

The putative protein sequence of the btd-like gene (Fig. 1A) reveals an SP1-like zinc finger (dark green) and buttonhead (light green) domain. Thus, btd-like gene is a member of the SP1 family of transcription factors, such as SP1 and SP4 in mouse and human, D-SP1 and BTD in Drosophila (Wimmer et al. 1993, 1996; Schöck et al. 1999a), and BTS-1 in zebra fish (Tallafuss et al. 2001). The highly conserved zinc finger domain $1>90 \%$ sequence identity) is insufficient to reconstruct the evolutionary relationship of the btd-like gene to other members of this gene family. Therefore, a comparison of the entire proteins was undertaken. As shown in Figure 1B, the putative protein contains a serine/threonine domain (red) followed by an alanine-rich sequence (yellow) similar to D-SP1 and BTD. It also contains a glycine-rich domain (blue), which is found in D-SP1, the paralog of BTD. In contrast, the glutamine-rich sequence (purple) found in BTD, D-SP1, SP1, SP4, and BTS1 is absent in the putative protein. This domain in BTD and D-SP1 cannot account for an evolutionary link to BTS1, as this se- 


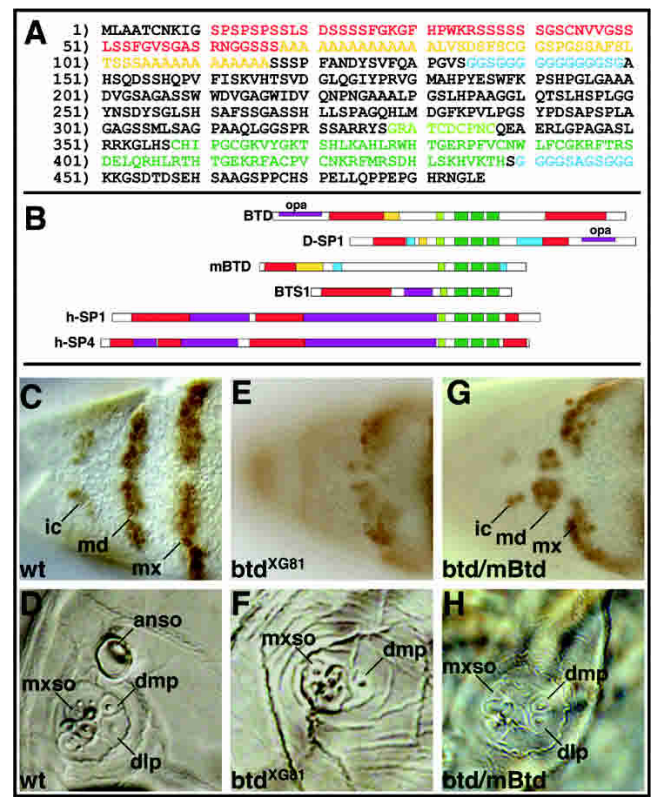

Figure 1. Cloning of $m B t d$ and rescue experiment in Drosophila. (A) Complete amino acid sequence of the mBtd protein. Functional domains are highlighted in different colors. $(B)$ Schematic comparison of the protein structure of the BTD, D-SP1, mBTD, BTS1, SP1, and SP4 proteins. Domains shown are as follows: zinc finger (dark green); btd domain (light green); serine/threonine-rich (red); alaninerich (yellow); glycine-rich (blue); glutamine-rich (purple). (opa) Glutamine-rich opa-elements. $(C-H) \mathrm{mBtd}$ rescues btd-dependent aspects of Drosophila embryo head development. $(C)$ Dorsal view of head segments in wild-type embryos visualized by anti-Engrailed antibody staining. The intercalary (ic), mandibular (md), and maxillary $(\mathrm{mx})$ stripes are visible. (D) Antennomaxillary complex of a wild-type larva. Note the maxillary sense organ (mxso) of the maxillary segment, the dorsolateral papilla (dlp) of the intercalary segment, the antennal sense organ (anso) of the antennal origin, and the dorsomedial papilla (dmp) of the ocular (oc) segment. (E) btd $d^{X G 81}$ mutant embryo lacking the antennal, intercalary, and mandibular segments (anti-Engrailed antibody staining). (F) In $b t d^{X G 81}$ mutant larvae, corresponding sense organs are absent. $(G) b t d^{X G 81}$ mutant embryos bearing one copy of the btd- $m B t d$ transgene show rescue of mandibular and intercalary segments. $(H) b t d^{X G 81}$ mutant larva showing the corresponding sensory organs. Anterior is to the left.

quence rather represents a typical opa-element, which is found in nonhomologous genes in Drosophila. Accordingly, we propose that Bts1 is an ortholog of Sp1 and Sp4, whereas the newly identified gene represents an ortholog of $D-S p 1$ and $b t d$.

To test in vivo for evolutionary conserved functions of btd homologs, different genes were expressed under the control of the regulatory region of the btd gene (Schöck et al. 1999a,b) in btd mutant Drosophila embryos. In these rescue experiments, the two previously identified btd-like genes $S p 1$ and Bts1 failed to provide any scorable rescuing activity anterior to the mandibular segment (Schöck et al. 1999b; Tallafuss et al. 2001). The expression of one copy of the newly identified gene resulted in the development of $b t d$-dependent mandibular $(100 \%)$ and, in addition, intercalary segments $(-10 \%$, $n>50$; Fig. $1 \mathrm{C}-\mathrm{H})$. Therefore, the encoded protein contains functional features of BTD, which are absent from SP1 and BTS1. In analogy to the Drosophila gene btd, we termed the gene mouse Btd $(\mathrm{mBt})$.

\section{mBtd expression pattern during mouse embryogenesis}

The spatiotemporal aspects of mBtd expression were analyzed by whole-mount in situ hybridization. The results of these experiments are summarized in Figure 2.

Initial expression of mBtd is found between embryonic day 7.0 (E7.0) and E7.5 of gestation, with a strong hybridization signal during gastrulation in embryonic ectoderm and primitive streak (Fig. 2A). During secondary gastrulation, transcripts become restricted to the tail bud (Fig. 2D). During organogenesis, mBtd mRNA appears within the central nervous system (CNS) in the telencephalon, midbrain-hindbrain boundary (MHB), spinal cord, otic vesicles, and nasal placodes (Fig. 2B,D,G).

Outside of the CNS, mBtd activity is restricted to the limbs. It is initially observed in the entire ectoderm of the limb anlagen at E9.5, with a more abundant expression in the ventral part (Fig. 2C,F). The expression becomes progressively more prominent in the AER and is additionally detected as a clear but less prominent signal in the ventral ectoderm (Fig. 2E,G-I).

These patterns suggest a possible role of $m B t d$ during gastrulation and during CNS and limb development.

\section{Loss of mBtd activity causes defects at the sites of expression}

To elucidate the role of $m B t d$ during mouse embryogenesis, we performed knockout experiments using a ho-

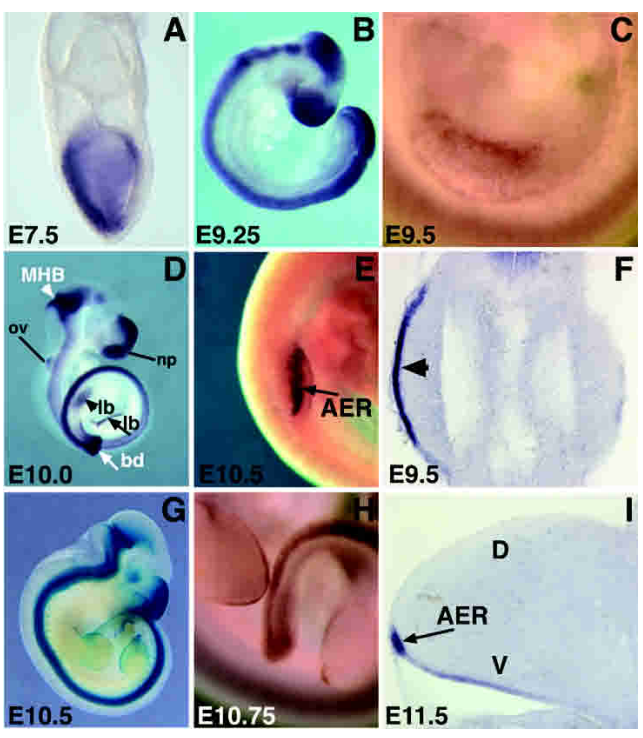

Figure 2. A highly restricted expression pattern of $m B t d$ in mouse embryogenesis. Whole-mount in situ hybridization $(A, B, C, D, E, H)$, LacZ staining $(G)$, and vibratome sections of whole-mount staining $(30 \mu \mathrm{m} ; F, I)$ presenting the expression of $m B t d$ at different stages of development. $m B t d$ is detected during gastrulation in the embryonic ectoderm and primitive streak $(A)$. Subsequently, $m B t d$ is found in the developing neural tube, in the prospective telencephalon, midbrain-hindbrain boundary, and spinal cord $(B, D, G)$. Expression is also obvious in the otic vesicle, in the nasal placode, and tail bud $(D)$. In the limb bud $m B t d$ is shown at E9.5 in the entire ecto$\operatorname{derm}(C, F)$ and at E10.5, E10.75, and E11.0 in the AER $(E, G, H, I)$ and ventral ectoderm $(I)$. In $C$ and $H$, anterior is to the left. In $F$, dorsal and anterior are to the top. In $I$, cross-section is at the level of the hindlimb. Arrows and arrowheads indicate expression domains. (D) Dorsal; (AER) apical ectodermal ridge; (bd) tail bud; (lb) limb bud; (MHB) midbrain-hindbrain boundary; (np) nasal placode; (ov) otic vesicle; (V) ventral. 
mologous recombination approach in which the coding region of $\mathrm{mBtd}$ was replaced by a bacterial lacZ gene in embryonic stem cells according to Mansouri (2001; details in Supplementary Fig. 1A-C). Heterozygous mBtd mutant individuals develop to term. About one-third of them exhibit a slight kink at the tip of the tail (Supplementary Fig. 1E), suggesting that a reduction of the $m B t d$ gene dose impairs posterior vertebra development at low but significant penetrance. Homozygous $m B t d$-deficient embryos also develop to term and die either during birth or immediately thereafter.

Morphological and histological inspection of the homozygous mBtd mutant individuals revealed multiple and severe developmental defects in locations where the gene normally is expressed. Exencephaly at the level of the forebrain, an acerebellar hindbrain, spina bifida, and loss of nasal and palate structures were observed in these embryos (Fig. 3I; data not shown). Most striking were truncations of the posterior axis and a reduction of both fore- and hindlimbs (Fig. 3B,D,I,K). To elucidate mBtd function and to explore the genetic network in which $m B t d$ is involved, we focused our attention on its role in limb development.

\section{mBtd maintains signaling in the developing limb}

The truncations of both fore- and hindlimbs of mutant embryos are variable, as exemplified by the range of E17.5 skeletal phenotypes of the mutants depicted in Figure $3 \mathrm{~B}$ and D. In most cases humerus and femur, although malformed, are generated, whereas the more distal parts of the limbs are variably affected (Fig. 3B,D; see legend for details). This phenotype is reminiscent of de-

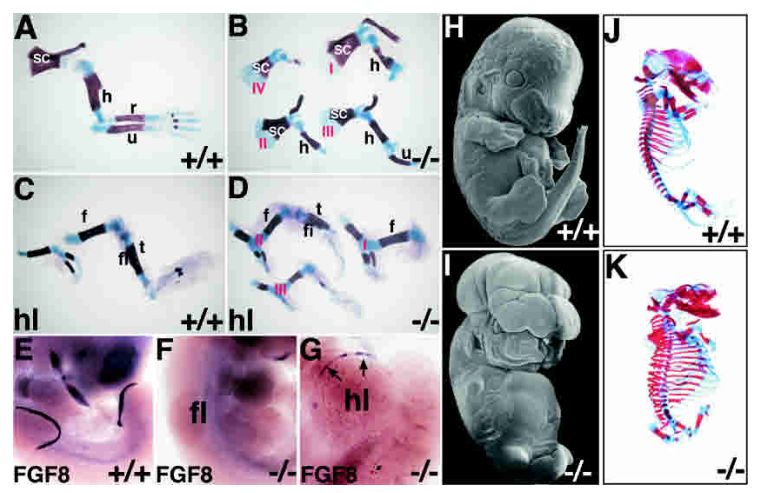

Figure 3. Severe truncations of mBtd-deficient limbs. Skeletal preparations at E17.5 show the severity and variability of the defects observed in the mutant limbs. Control fore- and hindlimbs are shown in $A$ and $C$, respectively. Several limb truncations are presented for the fore- and hindlimbs in $B$ and $D$. Some of these defects are shown, and the frequency, how often these or similar anomalies are observed. For the forelimbs: (I) 30/51; (II) $8 / 51$; (III) $12 / 51$; (IV) 1 . For the hindlimbs (I) 20/51; (II) 7/51; (III) 24/51. The numbers label individual limb variations. Whole skeletal preparations (E17.5) of control $(J)$ and mutant $(K)$ embryos showing the malformations in the trunk. The expression of Fgf8 in the AER is shown at E10.5 for control $(E)$ and mutant $(F, G)$ whole-mount embryos. It is obvious that at this stage of development, Fgf8 expression is already abolished in the forelimb ( $F$, five embryos tested), whereas a patchy expression domain is still recognizable in the hindlimb $(G$, five embryos). $H$ and $I$ represent scanning electron microscope of whole embryos at E12.5 from wild-type $(H)$ and $m B t d^{-1-}$ embryos. The arrows in $G$ point to the patchy expression in the AER of the hindlimbs. (f) Femur; (fi) fibula; (fl) forelimb; (hl) hindlimb; (h) humerus; (r) radius; (sc) scapula; $(\mathrm{t})$ tibia; $(\mathrm{u})$ ulna. fects caused by a perturbed formation of the AER (Capdevila and Izpisúa Belmonte 2001; Tickle 2003). Therefore, we examined the expression of genes that are critically involved in signaling events during AER formation and its proper function.

Limb outgrowth induction is based on cross-talk between epidermal and mesenchymal tissue /Capdevila and Izpisúa Belmonte 2001; Dudley et al. 2002; Tickle 2003). It is mediated by Fgf8 and Fgf10 (Min et al. 1998; $\mathrm{Xu}$ et al. 1998; Sekine et al. 1999; Lewandowski et al. 2000; Moon and Capecchi 2000; Moon et al. 2000; Sun et al. 2000, 2002). In mBtd mutant embryos, Fgf8 expression is initiated but reduced in the AER area at E9.5 (Fig. $4 \mathrm{I}, \mathrm{J})$. In the AER of the hindlimbs of E10.0, Fgf8 transcripts are still detected as a patchy pattern (Fig. 3G). However, they are absent from the forelimb bud (Fig. 3F). Similarly, mesenchymal Fgf10 transcripts are normally expressed at E9.5, but they gradually disappear during subsequent development (Fig. 5I,J; data not shown). Furthermore, the expression of Fgf4, another member of the FGF gene family that is normally expressed in the AER area after stage E10, could not be detected in $\mathrm{mBtd}$ mutant embryos (Fig. 4K,L). These findings indicate that $m B t d$ is not required to initiate but is required to maintain the Fgf8 and Fgf10 expression. The results are consistent with the observation that the mBtd mutant limb defects are similar to a phenotype caused by the loss of $F g f 8$ and Fgf 4 activities. In the absence of Fgf8, the limb elements are present but malformed (Lewandowski et al. 2000; Moon and Capecchi 2000), and they are even wild type-like in Fgf4-deficient limbs (Moon et al. 2000; Sun et al. 2000). Loss of the combined Fgf8 and Fgf4 activities, however, causes more severe limb truncations than the absence of Fgf8 only (Sun et al. 2002), and the limb truncations closely resemble the $\mathrm{mBtd}$ loss-of-function mutant limb buds shown here (Fig. 3).

Limb outgrowth defects were shown to be associated with an altered expression of Sonic hedgehog (Shh; Yang et al. 1997; Chiang et al. 2001; Kraus et al. 2001) in the posterior distal mesenchyme. In mBtd mutant embryos, expression of the signaling molecule $S h h$ is properly initiated, but the transcripts are progressively lost (Fig. 5A,B), as observed with Fgf8 and Fgf10. This is likely due to a disruption of the reciprocal feedback loop between the AER and posterior mesenchyme (polarizing region), which involves $\mathrm{Fgf4}$ and Shh activities (Laufer et al. 1994; Niswander et al. 1994). This argument is supported by the altered expression of the BMP antagonist Gremlin, which relays the $S h h / F g f 4$ feedback loop (Zuniga et al. 1999; Te Welscher et al. 2002). In mBtd mutant limbs, Gremlin expression is initiated normally but not maintained (data not shown). Furthermore, expression of the limb mesenchyme marker genes Gli3, Gbx2, and Hoxd12 is initiated normally (Büscher et al. 1997; Herault et al. 1999), but their activities are progressively lost (Figs. 4E,F, 5C,D,G,H). Collectively, these findings establish that $m B t d$ is specifically required to maintain signaling gene activities during early stages of limb outgrowth.

$\mathrm{mBtd}$ is also required for the dorsoventral limb patterning

AER formation involves a regulatory loop connecting FGF and the Wnt/ $\beta$-catenin signaling activities (Kengaku et al. 1998; Galceran et al. 1999; Kawakami et al. 2001; 


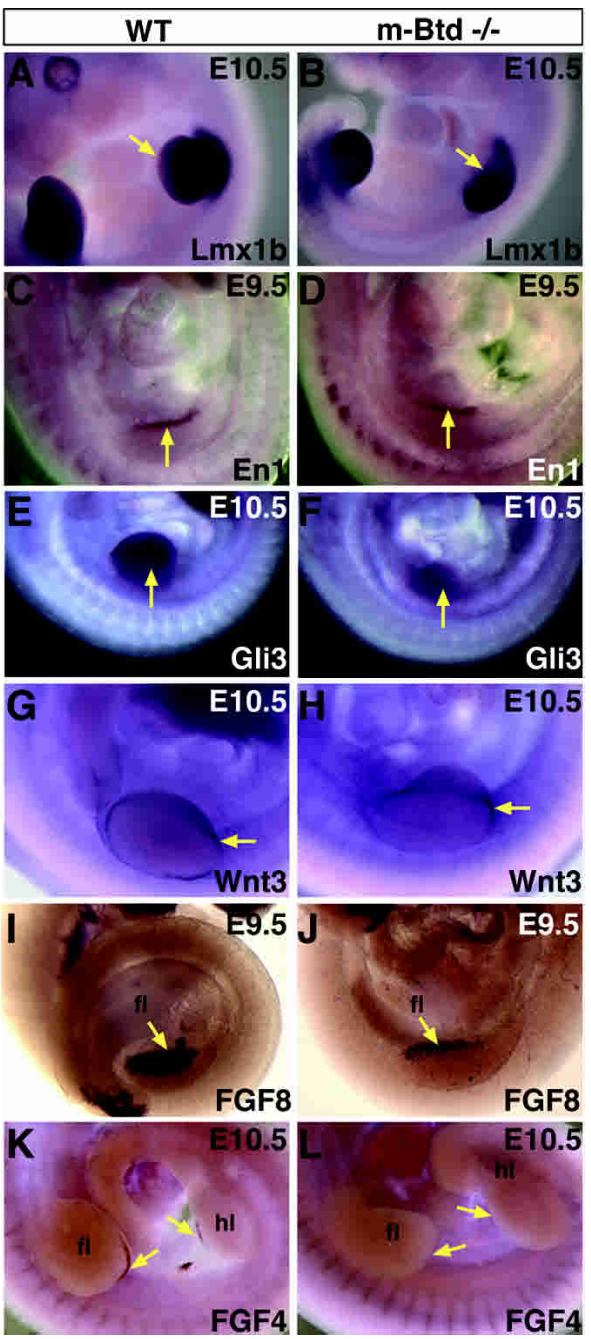

Figure 4. Analysis of the limb phenotype with molecular markers. Whole-mount in situ hybridization at E9.5 or E10.5 showing the expression pattern of molecular markers involved in limb patterning. $(I, J) F G F 8$ expression is detected in the forelimb of $\mathrm{E} 9.5 \mathrm{mBtd}^{-1}$ embryos, as compared with E10.5 in Figure 3F. $(K, L)$ The expression of Fgf4 is not initiated (10 embryos tested). $(G, H)$ The ectodermal Wnt3 expression is not altered by the loss of mBtd activity (five embryos tested). $(A, B)$ The expression domain of $L m \times 1 b$ in the forelimb is extended into the ventral portion of the bud (arrows; seven embryos tested), pointing to a progressive dorsalization of the limb. $(C, D)$ In the same embryo, the younger hindlimb still exhibits a normal dorsoventral expression pattern. Initial expression of En1 at E9.5 is normal. $(E, F)$ The expression of Gli3 is not maintained in $\mathrm{mBtd}^{-1-}$ limbs. Except for $A, B$, and $J$, rostral is to the left. Arrows indicate the expression domains in the limb.

Barrow et al. 2003). In chick limb mesenchyme, Wnt/ $\beta$ catenin signaling establishes Fgf10 expression, which in turn is necessary to initiate Wnt $3 a$ activity in the ectoderm. Subsequently, Wnt3a triggers Fgf8 expression in AER cells that maintains Fgf10 expression in a Wnt/ $\beta$ catenin-dependent manner in the mesenchyme (Kengaku et al. 1998; Kawakami et al. 2001). Mouse Wnt3 was also recently shown to be critical for AER formation and limb outgrowth, possibly as a part of the regulatory loop connecting the Wnt/ $\beta$-catenin and FGF signaling pathways as established for chick limb development (Barrow et al. 2003). This assignment fits the observation that lack of Wnt3 or $\beta$-catenin activities causes severe outgrowth defects in the developing mouse limb (Barrow et al. 2003). In both Wnt3 and $\beta$-catenin mutant limbs, expression of Shh, En1, and BMP4 is abolished and limbs become progressively dorsalized (Barrow et al. 2003). Furthermore, simultaneous inactivation of Lef1 and Tcf1, the mediators of the Wnt signaling pathway, abolishes En1 expression and prevents limb outgrowth (Galceran et al. 1999).

The limb truncations observed in mBtd mutants are similar to those seen in Wnt3 or $\beta$-catenin mutants. In particular, the expression of $L m x 1 b$, a marker for the dorsal limb mesenchyme, is extended ventrally, as has been observed in Wnt3 mutant limbs (Fig. 4A,B). In contrast to Wnt3 or $\beta$-catenin mutants, the expression of genes involved in dorsal ventral patterning, such as En1

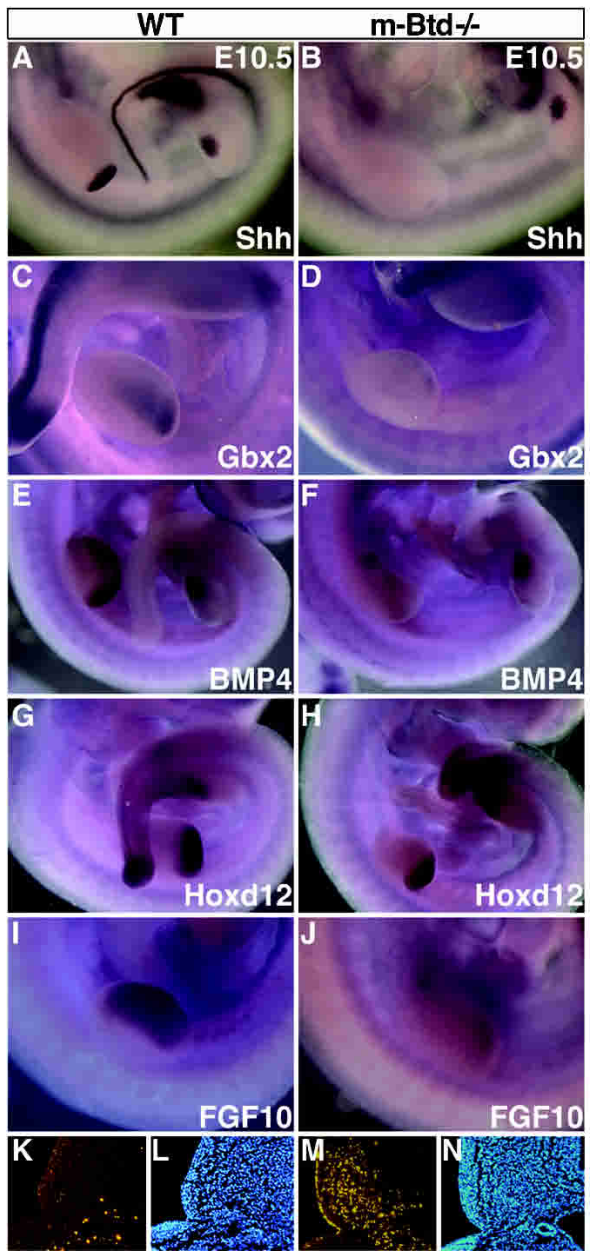

Figure 5. Expression of molecular markers and cell death analysis in the mBtd mutant limbs. $(A, B, E, F, I, J)$ Sonic Hedgehog, BMP, and FGF signaling is not maintained in $m$-Btd mutant limbs shown in whole-mount in situ at E10.5. Two other markers are also affected: Gbx2 $(C, D)$ and Hoxd12 $(G, H)$. In $A-I$, anterior is to the left. Apoptotic cell death was assayed on cross-sections of E10.5 embryos at the level of the forelimb bud by using TUNEL. $(K, M)$ Cell death in the forelimb bud of E10.5 control and mutant embryos is shown. $(M)$ Apoptosis is detected in the mesenchyme and ectoderm of the mBtd-deficient forelimb bud at E10.5. (K) There is little or no apoptosis in the control forelimb bud. $L$ and $N$ indicate the corresponding DAPI staining to $K$ and $M$, respectively. In $K-N$, dorsal is to the left. 
and BMP4 (Loomis et al. 1998; Ahn et al. 2001; Pizette et al. 2001), is normal in $m B t d$-deficient limbs, but is lost during development (Figs. 4C,D, 5E,F). This suggests that $m B t d$ acts downstream of Wnt3- $\beta$-catenin signaling, implying that Wnt3 expression is not affected in the ectoderm of $m B t d$-deficient limbs. Figure $4 \mathrm{G}$ and $\mathrm{H}$ shows that this is indeed the case. Moreover, apoptotic cell death shown to occur in Wnt3 mutant limbs (Barrow et al. 2003) can also be detected in dorsal mesenchyme and ectoderm of the forelimbs in $\mathrm{mBtd}$ lack-of-function individuals at E10.5 (Fig. 5K-N). However, cell death also occurs in limbs lacking FGF8 or FGF8 and FGF4 function (Moon and Capecchi 2000; Sun et al. 2002). Altogether, this indicates that like in the absence of Wnt3 and $\beta$-catenin signaling, the lack of $\mathrm{mBtd}$ function causes limbs to acquire a progressively dorsalized phenotype. Our results suggest that $m B t d$ may maintain the expression of genes acting downstream of Wnt/ $\beta$-catenin signaling in the limb. However, we cannot exclude the possibility that the observed dorsalization in $\mathrm{mBtd}$ mutant limbs is related to the progressive loss of the AER. Mechanisms regulating AER formation may be linked to those establishing dorsoventral patterning (Chen and Johnson 1999). Accordingly, although our findings suggest that $\mathrm{mBtd}$ is required for the initiation of FGF4 expression in the AER, it may rather reflect the loss of AER integrity. We conclude that $m B t d$ is specifically required to maintain signaling gene activities during early stages of limb outgrowth.

\section{Does lack of mBtd activity perturb patterning and outgrowth at all sites of embryonic expression?}

We have not addressed the role of $M B T D$ during the development of the tail bud, midbrain-hindbrain boundary, and nasal structures. However, it appears likely that in all these tissues with related organizer functions bearing Fgf8 expression, $\mathrm{mBt}$ is necessary to maintain these critical intercellular signaling events during embryogenesis, as suggested by its role in limb development. Clearly, this hypothesis needs to be addressed in the future.

\section{Materials and methods}

Cloning of mouse mBtd $c D N A$

Using degenerated primers as described (Treichel et al. 2001), a 230-bp PCR product was amplified from mouse genomic DNA and identified by hybridization to Drosophila buttonhead probe. This fragment was used to screen an E8.5 mouse cDNA library (kindly provided by B. Hogan, Vanderbilt University Medical Center, Nashville, TN), from which several clones were isolated and further selected by whole-mount in situ hybridization. Five overlapping clones were used to assemble the fulllength cDNA sequence (complete amino acid sequence shown in Fig. 1).

Gene targeting and chimeric mice generation

Gene targeting and chimeric mice generation were performed as described (Mansouri 2001). Targeted clones were confirmed by $5^{\prime}$ and $3^{\prime}$ external probes (Supplementary Fig. 1). One targeted clone produced germline chimeras. All experiments were performed on a mixed (NMRIx129Sv) background. Mice and embryos were genotyped either by genomic Southern blot or PCR.

In situ hybridization, lacZ-staining, histology, immunohistochemistry, TUNEL assay, and skeletal preparations

In situ hybridizations using digoxygenin were performed as described (Wilkinson 1992). For whole-mount in situ hybridization, a minimum of five mutant embryos was used for the analysis. For histological analysis, embryos were fixed in 4\% PFA, and paraffin sections $(10 \mu \mathrm{m})$ were prepared. Immunological staining of Drosophila embryos was performed using as primary antibodies anti- $\beta$-galactosidase (Cappel) and 4D9 antiEngrailed/Invected together with the Vectastain ABC Elite Kit (Vector). To identify homozygous mutant embryos, blue balancers were used.

\section{Drosophila strains}

We used Oregon R as wild type, btdXG81, svbYP17b btdXG81, and a homozygous line of the btd-mBtd transgene. The $s v b$ btd double mutant was used to identify btd mutant cuticles (Wimmer et al. 1993, 1996). Apoptosis assays were performed using the Apoptag kit (Invitrogen) according to the manufacturer's recommendations.

\section{Acknowledgments}

We thank J. Krull and S. Mahsur for excellent technical assistance, R. Altschäffel for excellent photographic work, and G. Goudreau and P. Collombat for help with the figures. The input of the mouse house crew and in particular C. Zeden, R. Libal, and U. Teichmann are highly acknowledged. We thank A.P. McMahon, R. Harland, D. Duboule, A. Joyner, G. Martin, B. Hogan, and P. Burbach for probes. This work was supported by the European community QLG3-CT-2000-01625 and BMBF 0311947. 4D9 was obtained from the Developmental Studies Hybridoma Bank (University of Iowa).

The publication costs of this article were defrayed in part by payment of page charges. This article must therefore be hereby marked "advertisement" in accordance with 18 USC section 1734 solely to indicate this fact.

\section{References}

Ahn, K., Mishina, Y., Hanks, M.C., Behringer, R.R., and Crenshaw III, E.B. 2001. BMPR-IA signaling is required for the formation of the apical ectodermal ridge and dorsal-ventral patterning of the limb. Development 128: 4449-4461.

Barrow, J.R., Thomas, K.R., Boussadia-Zahui, O., Moore, R., Kemler, R., Capecchi, M.R., and McMahon, A.P. 2003. Ectodermal Wnt3/ $\beta$ catenin signaling is required for the establishment and maintenance of the apical ectodermal ridge. Genes \& Dev. 17: 394-409.

Buscher, D., Bosse, B., Heymer, J., and Ruther, U. 1997. Evidence for genetic control of Sonic hedgehog by Gli3 in mouse limb development. Mech. Dev. 62: 175-182.

Capdevila, J. and Izpisúa Belmonte, J.C. 2001. Patterning mechanisms controlling vertebrate limb development. Annu. Rev. Cell. Dev. Biol. 17: $87-132$.

Chen, H. and Johnson, R.L. 1999. Dorsoventral patterning of the vertebrate limb: A process governed by multiple events. Cell Tissue Res. 296: 67-73.

Chiang, C., Litingtung, Y., Harris, M.P., Simandl, B.K., Li, Y., Beachy, P.A., and Fallon, J.F. 2001. Manifestation of the limb prepattern: Limb development in the absence of sonic hedgehog function. Dev. Biol. 236: 421-435.

Cohen, S. and Jürgens, G. 1991. Drosophila headlines. Trends Genet. 7: 267-272.

Dudley, A.T., Ros, M.A., and Tabin, C.J. 2002. A re-examination of proximodistal patterning during vertebrate limb development. Nature 418: $539-544$.

Finkelstein, R. and Boncinelli, E. 1994. From fly head to mammalian forebrain: The story of otd and Otx. Trends Genet. 10: 310-315.

Galceran, J., Farinas, I., Depew, M.J., Clevers, H., and Grosschedl, R. 1999. Wnt3 $\mathrm{a}^{-/-}$-like phenotype and limb deficiency in Lef1 ${ }^{-/-} \mathrm{Tcf}^{-/-}$ mice. Genes \& Dev. 13: 709-717.

Herault, Y., Beckers, J., Gerard, M., and Duboule, D. 1999. Hox gene expression in limbs: Colinearity by opposite regulatory controls. Dev. Biol. 208: 157-165.

Kawakami, Y., Capdevila, J., Buscher, D., Itoh, T., Rodriguez Esteban, C., and Izpisua Belmonte, J.C. 2001. WNT signals control FGF-dependent limb initiation and AER induction in the chick embryo. Cell 104: 891-900.

Kengaku, M., Capdevila, J., Rodriguez-Esteban, C., De La Pena, J., Johnson, R.L., Izpisua Belmonte, J.C., and Tabin, C.J. 1998. Distinct WNT pathways regulating AER formation and dorsoventral polarity in the chick limb bud. Science 280: 1274-1277. 
Kraus, P., Fraidenraich, D., and Loomis, C.A. 2001. Some distal limb structures develop in mice lacking Sonic hedgehog signaling. Mech. Dev. 100: 45-58.

Laufer, E., Nelson, C.E., Johnson, R.L., Morgan, B.A., and Tabin, C. 1994. Sonic hedgehog and Fgf-4 act through a signaling cascade and feedback loop to integrate growth and patterning of the developing limb bud. Cell 79: 993-1003.

Lewandoski, M., Sun, X., and Martin, G.R. 2000. Fgf8 signaling from the AER is essential for normal limb development. Nat. Genet. 26: 460463.

Loomis, C.A., Kimmel, R.A., Tong, C.X., Michaud, J., and Joyner, A.L. 1998. Analysis of the genetic pathway leading to formation of ectopic apical ectodermal ridges in mouse Engrailed-1 mutant limbs. Development 125: 1137-1148.

Mansouri, A. 2001. Determination of gene function by homologous recombination using embryonic stem cells and knockout mice. Methods Mol. Biol. 175: 397-413.

Min, H., Danilenko, D.M., Scully, S.A., Bolon, B., Ring, B.D., Tarpley, J.E., DeRose, M., and Simonet, W.S. 1998. Fgf-10 is required for both limb and lung development and exhibits striking functional similarity to Drosophila branchless. Genes \& Dev. 12: 3156-3161.

Moon, A.M. and Capecchi, M.R. 2000. Fgf8 is required for outgrowth and patterning of the limbs. Nat. Genet. 26: 455-459.

Moon, A.M., Boulet, A.M., and Capecchi, M.R. 2000. Normal limb development in conditional mutants of Fgf4. Development 127: 989996.

Niswander, L., Jeffrey, S., Martin, G.R., and Tickle, C. 1994. A positive feedback loop coordinates growth and patterning in the vertebrate limb. Nature 371: 609-612.

Pizette, S., Abate-Shen, C., and Niswander, L. 2001. BMP controls proximodistal outgrowth, via induction of the apical ectodermal ridge, and dorsoventral patterning in the vertebrate limb. Development 128: 4463-4474.

Schöck, F., Sauer, F., Jäckle, H., and Purnell, B.A. 1999a. Drosophila head segmentation factor buttonhead interacts with the same TATA boxbinding protein-associated factors and in vivo DNA targets as human Sp1 but executes a different biological program. Proc. Natl. Acad. Sci. 96: 5061-5065.

Schöck, F., Purnell, B.A., Wimmer, E.A., and Jäckle, H. 1999b. Common and diverged functions of the Drosophila gene pair D-Sp1 and buttonhead. Mech. Dev. 89: 125-132.

Sekine, K., Ohuchi, H., Fujiwara, M., Yamasaki, M., Yoshizawa, I., Sato, T., Yagishita, N., Matsui, D., Koga, Y., Itoh, N., et al. 1999. Fgf10 is essential for limb and lung formation. Nat. Genet. 21: 138-141.

Simeone, A. 2002. Towards the comprehension of genetic mechanisms controlling brain morphogenesis. Trends Neurosci. 25: 119-121.

Sun, X., Lewandoski, M., Meyers, E.N., Liu, Y.H., Maxson Jr., R.E., and Martin, G.R. 2000. Conditional inactivation of Fgf4 reveals complexity of signalling during limb bud development. Nat. Genet. 25: 83-86.

Sun, X., Mariani, F.V., and Martin, G.R. 2002. Functions of FGF signalling from the apical ectodermal ridge in limb development. Nature 418: $501-508$.

Tallafuss, A., Wilm, T.P., Crozatier, M., Pfeffer, P., Wassef, M., and BallyCuif, L. 2001. The zebrafish buttonhead-like factor Bts1 is an early regulator of pax2.1 expression during midhindbrain development. Development 128: 4021-4034.

Te Welscher, P., Zuniga, A., Kuijper, S., Drenth, T., Goedemans, H.J., Meijlink, F., and Zeller, R. 2002. Progression of vertebrate limb development through SHH-mediated counteraction of GLI3. Science 298: $827-830$.

Tickle, C. 2003. Patterning systems-From one end of the limb to the other. Dev. Cell 4: 449-458.

Treichel, D., Becker, M.B., and Gruss, P. 2001. The novel transcription factor Sp5 exhibits a dynamic and highly restricted expression pattern during mouse embryogenesis. Mech. Dev. 101: 175-179.

Wilkinson, D.G. 1992. In situ hybridisation: A practical approach, pp. 75-84. Oxford University Press, London.

Wimmer, E.A., Jäckle, H., Pfeifle, C., and Cohen, S.M. 1993. A Drosophila homologue of human $S p 1$ is a head-specific segmentation gene. Nature 366: 690-694.

Wimmer, E.A., Frommer, G., Purnell, B.A., and Jäckle, H. 1996. Buttonhead and D-Sp1: A novel Drosophila gene pair. Mech. Dev. 59: 53-62.

Xu, X., Weinstein, M., Li, C., Naski, M., Cohen, R.I., Ornitz, D.M., Leder,
P., and Deng, C. 1998. Fibroblast growth factor receptor 2 (FGFR2)mediated reciprocal regulation loop between FGF8 and FGF10 is essential for limb induction. Development 125: 753-765.

Yang, Y., Drossopoulou, G., Chuang, P.T., Duprez, D., Marti, E., Bumcrot, D., Vargesson, N., Clarke, J., Niswander, L., McMahon, A., et al. 1997. Relationship between dose, distance and time in Sonic Hedgehog-mediated regulation of anteroposterior polarity in the chick limb. Development 124: 4393-4404.

Zuniga, A., Haramis, A.P., McMahon, A.P., and Zeller, R. 1999. Signal relay by BMP antagonism controls the SHH/FGF4 feedback loop in vertebrate limb buds. Nature 401: 598-602. 


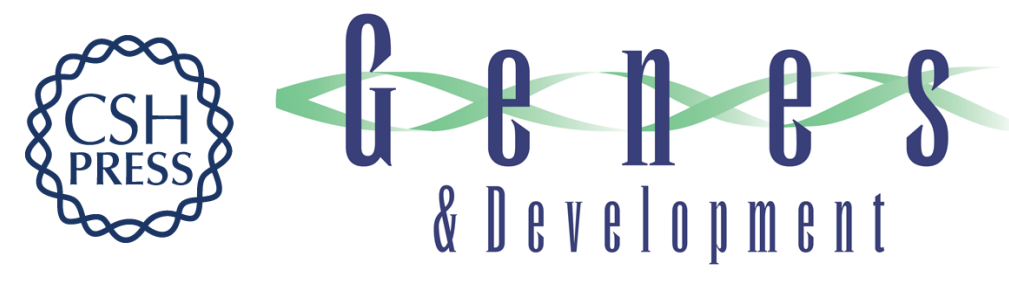

\section{$m B t d$ is required to maintain signaling during murine limb development}

Dieter Treichel, Frieder Schöck, Herbert Jäckle, et al.

Genes Dev. 2003, 17:

Access the most recent version at doi:10.1101/gad.274103

\section{Supplemental http://genesdev.cshlp.org/content/suppl/2003/10/09/17.21.2630.DC1 Material}

References This article cites 35 articles, 10 of which can be accessed free at: http://genesdev.cshlp.org/content/17/21/2630.full.html\#ref-list-1

\section{License}

Email Alerting

Receive free email alerts when new articles cite this article - sign up in the box at the top Service 